\title{
Characterizations of exponentiality within the HNBUE class and related tests
}

\author{
José R. Berrendero and Javier Cárcamo * \\ Departamento de Matemáticas, Universidad Autónoma de Madrid, 28049 Madrid
}

\begin{abstract}
A harmonic new better than used in expectation (HNBUE) variable is a random variable which is dominated by an exponential distribution in the convex stochastic order. We use a recently obtained condition on stochastic equality under convex domination to derive characterizations of the exponential distribution and bounds for HNBUE variables based on the mean values of the order statistics of the variable. We apply the results to generate discrepancy measures to test if a random variable is exponential against the alternative that is HNBUE, but not exponential.
\end{abstract}

Keywords: Convex order; order statistics; HNBUE distributions; hypothesis testing. $M S C: 60 \mathrm{E} 15 ; 60 \mathrm{E} 99 ; 62 \mathrm{E} 99 ; 62 \mathrm{G} 10 ; 62 \mathrm{E} 20 ;$ secondary $62 \mathrm{~N} 05$

*Research supported by the Spanish MEC, grants MTM2007-66632 and MTM2008-06281C02-02, and Comunidad de Madrid, grant S-0505/ESP/0158.

E-mail addresses: joser.berrendero@uam.es and javier.carcamo@uam.es 


\section{Introduction}

Stochastic orderings have important applications in diverse fields. For instance, several notions of stochastic orders have been extensively used in Economics to compare risks of alternative investments or income levels in different populations. Stochastic dominance rules of convex (or concave) type are specially useful in the analysis of poverty and income inequality (see for instance [10]).

Partial order relationships among random variables also play a fundamental role in reliability theory, where several orderings have been introduced to compare lifetime distributions, which is useful in the analysis of systems reliability. Moreover, stochastic orders are also behind several concepts of aging since important classes of distributions can be defined by comparing, in different orderings, a lifetime variable (or its remaining time at age $t$ ) with the exponential distribution, which satisfies the non-ageing property. We also refer the interested reader to the books $[12 ; 13]$ for a comprehensive treatment on stochastic orders and its multiple applications.

We consider the class of distributions with the harmonic new better than used in expectation (HNBUE) (resp. harmonic new worse than used in expectation (HNWUE)) property. These two classes of random variables are defined by means of the comparison with the exponential distribution with respect to the convex order. We recall that given two integrable random variables $X$ and $Y$, we say that $X$ is less or equal $Y$ in the convex order, and we write $X \leq_{c x} Y$, if $\operatorname{E} f(X) \leq \operatorname{E} f(Y)$, for every convex function $f: \mathbb{R} \rightarrow \mathbb{R}$ for which the previous expectations are well defined. A nonnegative variable $X$ is said to be HNBUE (resp. HNWUE) if $X \leq_{c x} Y$ (resp. $Y \leq_{c x} X$ ), where $Y$ is an exponential random variable.

The class of variables HNBUE (and its dual HNWUE) is a fairly large class of random variables in reliability theory which contains the class of NBUE (new better than used in expectation) distributions and, in consequence, it includes all IFR (increasing failure rate), IFRA (increasing failure rate average) and NBU (new better than used) distributions. See [2] for a detailed study of these classes of lifetime distributions.

In this paper we use a specific result in [4] on stochastic equality under convex- 
type domination to derive characterizations of the exponential distribution in the classes of HNBUE and HNWUE variables. At the same time, we obtain bounds for some functions of the expected value of the order statistics of random variables with these properties. These results can in turn be applied to define simple discrepancy measures (with a precise interpretation in terms of the expected system lifetime of series and parallel systems) which are useful to test the null hypothesis that a random variable follows an exponential distribution against the alternative that it follows a HNBUE (or HNWUE) distribution.

The paper is structured as follows: In next section we obtain some characterizations of the exponential distribution and derive bounds on certain functions of the mean order statistics for HNBUE (or HNWUE) variables. Testing of exponentiality against HNBUE or HNWUE alternatives is discussed in Section 3. All the proofs are included in a final appendix.

\section{Bounds and characterizations}

Let $X$ and $Y$ be two integrable random variables taking values on $\mathbb{R}$ with distribution functions $F$ and $G$, respectively. We denote by $F^{-1}$ and $G^{-1}$ the respective quantile functions, i.e.,

$$
F^{-1}(t):=\inf \{x: F(x) \geq t\}, \quad 0<t<1 .
$$

For a measurable real function $\omega$ on $[0,1]$, we define

$$
\Delta_{\omega}(X, Y):=\int_{0}^{1}\left(G^{-1}(t)-F^{-1}(t)\right) \omega(t) \mathrm{d} t,
$$

whenever the above integral exists. The integral in (1) can be interpreted as a weighted and signed Wasserstein distance between the variables $X$ and $Y$.

In what follows $\mathcal{I}$ denotes the class of non decreasing real functions on $[0,1]$ and $\mathcal{I}^{*}$ stands for the subclass of strictly increasing functions of $\mathcal{I}$. We also use the notation $X={ }_{s t} Y$ to indicate the equality in distribution of $X$ and $Y$.

Next result (stated and proved in [4]) establishes a characterization of the convex order through the $\Delta$-distance defined above and a simple condition guaranteeing the equality in distribution of two ordered random variables: 
Theorem 1. Let $X$ and $Y$ be two integrable random variables. We have:

(a) $X \leq_{c x} Y$ if and only if $\Delta_{\omega}(X, Y) \geq 0$, for all $\omega \in \mathcal{I}$.

(b) If $X \leq_{c x} Y$ and $\Delta_{\omega}(X, Y)=0$ for some $\omega \in \mathcal{I}^{*}$, then $X={ }_{s t} Y$.

We shall show that the $\Delta$-distance (1) used in Theorem 1 is closely connected with the mean value of certain functions of the order statistics. Before applying the last theorem to obtain characterizations of exponentiality we need to introduce some notation. Given an integrable random variable $X$, for $k \geq 1$, let $\boldsymbol{X}_{k}:=$ $\left(X_{1}, \ldots, X_{k}\right)$ be a random sample of $X$. We denote by $X_{i: k}$ the $i$-th order statistics $(1 \leq i \leq k)$ of the random vector $\boldsymbol{X}_{k}$. For $k \geq 1$ and $0 \leq m \leq k$, let us denote by $S_{m: k}(X)$ (resp. $\left.s_{m: k}(X)\right)$ the expectation of the sum of the $m$ greatest (resp. smallest) order statistics of $\boldsymbol{X}_{k}$, i.e.,

$$
S_{m: k}(X):=\sum_{i=k+1-m}^{k} \mathrm{E} X_{i: k}, \quad s_{m: k}(X):=\sum_{i=1}^{m} \mathrm{E} X_{i: k},
$$

with the convention $\sum_{i=k+1}^{k}=\sum_{i=1}^{0}=0$. If the variable $X$ measures the lifetime of certain item, the function $S_{m: k}(X)$ (resp., $s_{m: k}(X)$ ) is nothing but the sum of the expected lifetime of the $m$ items with longest (resp., shortest) life out of a random sample of size $k$ of $X$. In particular, $S_{1: k}(X)=\mathrm{E} X_{k: k}$ (resp., $s_{1: k}(X)=\mathrm{E} X_{1: k}$ ) is the expected system lifetime of a parallel (resp., series) system with $k$ independent components distributed as $X$.

Let $\mu_{i: k}^{e}$ denote the mean of the $i$-th order statistics of a random sample of size $k$ of a variable with the exponential distribution with mean 1 , that is

$$
\mu_{i: k}^{e}=\sum_{r=1}^{i} \frac{1}{k+1-r}, \quad 0 \leq i \leq k .
$$

The following result is a consequence of Theorem 1 :

Proposition 1. Let $X$ be an integrable HNBUE or HNWUE random variable with mean $\mu$. The following conditions are equivalent:

(a) $X$ is exponential with mean $\mu$. 
(b) For some $k \geq 2$ and $1 \leq m<k$ or for some $p \geq 2$ and $1 \leq l<p$,

$$
S_{m: k}(X)-s_{l: p}(X)=\mu\left[m\left(1+\mu_{k-m: k}^{e}\right)-\left(l-(p-l) \mu_{l: p}^{e}\right)\right]
$$

where $\mu_{i: k}^{e}$ is given in (3).

On taking $m=0$ and $l=1$ in (b) of the last proposition, we obtain the condition $\mathrm{E}_{1: p}=\mu \mu_{1: p}^{e}(p \geq 2)$, which is the main result of Basu and Kirmani [3]. A generalization of [5, Theorem 4.1] can be given in an analogous way. Notice that this condition can also be written in terms of the so-called S-Gini index (see e.g. [15]) of a nonnegative random variable with positive mean defined by

$$
G_{p}(X):=1-\frac{\mathrm{E} X_{1: p}}{\mathrm{E} X}, \quad p \geq 2 .
$$

The S-Gini index provides information on the (normalized) difference between the mean lifetime and the expected system lifetime of a series system with $p$ independent components as $X$.

We can hence assert: If $X$ is HNBUE or HNWUE then $X$ has an exponential distribution if and only if $G_{p}(X)=1-1 / p$, for some $p \geq 2$. The case $p=2$ leads to the usual Gini coefficient

$$
G_{2}(X)=\frac{\mathrm{E}\left|X_{1}-X_{2}\right|}{2 \mathrm{E} X}=\frac{\mathrm{E}\left(X_{2: 2}-X_{1: 2}\right)}{2 \mathrm{E} X} .
$$

The other simplest choice, $m=1$ and $p=0$, generates a condition on the expected maxima $\mathrm{E} X_{k: k}=\mu \mu_{k: k}^{e}(k \geq 2)$. Putting together the two conditions we can also assert that the expected system lifetime of just one series (or parallel) system with $k \geq 2$ independent components whose lifetimes are distributed as a HNBUE or HNWUE variable $X$ can be used to detect whether $X$ is exponential or not.

By taking $p=k$ and $m=l=1$, we obtain that the expected range, $\mathrm{E}\left(X_{k: k}-\right.$ $\left.X_{1: k}\right)$, for any $k \geq 2$, can also be used to deduce if a distribution with the HNBUE or HNWUE property is exponential. In general, by selecting different values for $m, k, l, p$ in last proposition, it is possible to derive many new conditions that lead to the exponential distribution. 
The definition itself of a HNBUE variable $X$ implies that $\mathrm{E} \phi(X) \leq \mathrm{E} \phi(Y)$, where $Y$ is an exponential random variable with mean $\mathrm{E} X$ and $\phi$ is a convex function such that the expectations are well defined. However, in the context of reliability theory, instead of bounds for the expected value of functions of $X$, it is sometimes more important to have bounds for the expectation of the order statistics of random samples of $X$. The proof of the next proposition directly follows from the proof of the previous proposition and Theorem 1 (a).

Proposition 2. Let $X$ be a nonnegative HNBUE (respectively HNWUE) random variable with mean $\mu$. For all $k, p \geq 1,0 \leq m \leq k$ and $0 \leq l \leq p$, we have

$$
\begin{gathered}
S_{m: k}(X)-s_{l: p}(X) \leq \mu\left[m\left(1+\mu_{k-m: k}^{e}\right)-\left(l-(p-l) \mu_{l: p}^{e}\right)\right] \\
\left(\text { respectively } S_{m: k}(X)-s_{l: p}(X) \geq \mu\left[m\left(1+\mu_{k-m: k}^{e}\right)-\left(l-(p-l) \mu_{l: p}^{e}\right)\right]\right),
\end{gathered}
$$

where $\mu_{i: k}^{e}$ is given in (3).

\section{Tests for exponentiality against HNBUE alternatives}

\subsection{Discrepancy measures and critical regions}

If the variable $X$ measures the life length of certain unit, the results in Section 2 can be applied to generate measures of discrepancy to test the null hypothesis $H_{0}: X$ is an exponential variable, that is, the unit does not age with time, against the alternative $H_{1}: X$ is HNBUE (or HNWUE), but it does age with time. The discrepancies are obtained by selecting different values for $m, k, l$ and $p$ in Propositions 1 and 2 . Here, we discuss three possibilities which are relatively simple for analysis, provide a natural interpretation and give satisfactory results. The first one is a discrepancy based on the expected maximum out of $k$ observations:

$$
\Delta_{k, \max }:=\mu_{k: k}^{e}-\frac{\mathrm{E} X_{k: k}}{\mathrm{E} X}, \quad k \geq 2 .
$$

The second discrepancy focusses on the expected minimum instead of the expected maximum:

$$
\Delta_{k, \text { min }}:=\left(1-\mu_{1: k}^{e}\right)-\left(1-\frac{\mathrm{E} X_{1: k}}{\mathrm{E} X}\right)=\frac{\mathrm{E} X_{1: k}}{\mathrm{E} X}-\mu_{1: k}^{e}, \quad k \geq 2 .
$$


The case $k=2$ corresponds to the usual Gini index whereas larger values of $k$ correspond to the S-Gini index mentioned above. Finally, the third discrepancy takes into account the expected range over $k$ observations, and therefore uses the information contained by the two first discrepancies:

$$
\Delta_{k, \text { range }}:=\frac{\mu_{k: k}^{e}-\mu_{1: k}^{e}}{k}-\frac{\mathrm{E} X_{k: k}-\mathrm{E} X_{1: k}}{k \mathrm{E} X}=\frac{1}{k}\left(\Delta_{k, \max }+\Delta_{k, \min }\right), \quad k \geq 2 .
$$

Remark 1. It should be pointed out that, due to the strong dependency among the order statistics, we have

$$
\Delta_{2}:=\Delta_{2, \max }=\Delta_{2, \min }=\Delta_{2, \text { range }}=\Delta_{3, \text { range }}
$$

The first equalities above follow from $\mathrm{E} X_{1: 2}+\mathrm{E} X_{2: 2}=2 \mathrm{E} X$ while for the last one we use $2 \mathrm{E}\left(X_{3: 3}-X_{1: 3}\right)=3 \mathrm{E}\left(X_{2: 2}-X_{1: 2}\right)$. Therefore, the discrepancy $\Delta_{2}$ can be interpreted in 4 different ways: $\Delta_{2}$ measures the difference between the expected system lifetime of a series (or a parallel) system from $X$ with two independent components and the corresponding from an exponential variable. Furthermore, it can be also viewed as a quantity that gives information about the deviation from the exponentiality in the expected range length of two or three independent variables as $X$.

By the results of Section 2, the three discrepancies fulfill $\Delta_{k, \bullet}=0$ under the null hypothesis (exponentiality) and $\Delta_{k, \bullet}>0$ under the HNBUE alternative $\left(\Delta_{k, \bullet}<0\right.$ under the HNWUE alternative), where here, and hereafter, we use the notation $\Delta_{k, \bullet}$ to indicate any of the discrepancy measures described above $\left(\Delta_{k, \max }, \Delta_{k, \min }\right.$ and $\left.\Delta_{k, \text { range }}\right)$. Therefore, starting from a sample $X_{1}, \ldots, X_{n}$ of $n$ independent observations with the same distribution as $X$, it seems natural to estimate $\Delta_{k}$ • and reject $H_{0}$ whenever the estimate is large enough (in the case HNBUE) or small enough (in the case HNWUE).

The estimation of the discrepancies involves the estimation of the expected extreme order statistics $\mathrm{E} X_{1: k}$ and $\mathrm{E} X_{k: k}(k \geq 2)$. We adopt here a plug-in approach and use the estimators that result from computing the expectations under the empirical distribution $F_{n}$ corresponding to the sample. It turns out that the 
resulting estimators belong to the class of L-statistics:

$$
\mathrm{E}_{F_{n}}\left(X_{k: k}\right)=\sum_{i=1}^{n}\left[\left(\frac{i}{n}\right)^{k}-\left(\frac{i-1}{n}\right)^{k}\right] X_{i: n}
$$

and

$$
\mathrm{E}_{F_{n}}\left(X_{1: k}\right)=\sum_{i=1}^{n}\left[\left(1-\frac{i-1}{n}\right)^{k}-\left(1-\frac{i}{n}\right)^{k}\right] X_{i: n} .
$$

As a direct consequence, our tests are based on the following estimated discrepancies:

$$
\begin{gathered}
\hat{\Delta}_{k, \max }:=\mu_{k: k}^{e}-\frac{1}{\bar{X}} \sum_{i=1}^{n}\left[\left(\frac{i}{n}\right)^{k}-\left(\frac{i-1}{n}\right)^{k}\right] X_{i: n}, \\
\hat{\Delta}_{k, \min }:=\frac{1}{\bar{X}} \sum_{i=1}^{n}\left[\left(1-\frac{i-1}{n}\right)^{k}-\left(1-\frac{i}{n}\right)^{k}\right] X_{i: n}-\mu_{1: k}^{e},
\end{gathered}
$$

where $\bar{X}$ is the sample mean. Hence

$$
\hat{\Delta}_{k, \text { range }}:=k^{-1}\left(\hat{\Delta}_{k, \max }+\hat{\Delta}_{k, \text { min }}\right)
$$

Remark 2. The estimator of the quantity $\Delta_{2}$ defined in (5) is simply

$$
\hat{\Delta}_{2}:=\frac{3}{2}-\frac{2}{\bar{X}} \frac{1}{n^{2}} \sum_{i=1}^{n} i X_{i: n}+\frac{1}{n} .
$$

The statistics $\hat{\Delta}_{k \text {,max }}$ and $\hat{\Delta}_{k \text {,min }}$ are similar to those already proposed in [9] for the same testing problem. Moreover, the unbiased version of $\hat{\Delta}_{2}$ was analyzed in depth by Hendi et al in [6] where the exact distribution of this statistic is obtained. Our results give some insight by relating $\hat{\Delta}_{k \text {,max }}$ and $\hat{\Delta}_{k \text {,min }}$ with the mean order statistics, which has the advantage of providing them with a precise meaning in reliability theory.

The following proposition establishes the asymptotic normality of the estimated discrepancies under the null hypothesis and gives expressions for the corresponding asymptotic variances. We use the notation " $\longrightarrow d$ " to indicate "convergence in distribution".

Proposition 3. Assume that $H_{0}$ holds and that $n \rightarrow \infty$ and let $U$ be a random variable uniformly distributed on $(0,1)$. Then, 
(a) For all $k \geq 2, \sqrt{n} \hat{\Delta}_{k, \text { max }} \longrightarrow d \mathrm{~N}\left(0, \sigma_{k, \text { max }}^{2}\right)$, where

$$
\sigma_{k, \max }^{2}=\operatorname{Var}\left[\left(\mu_{k: k}^{e}-k U^{k-1}\right) \log (1-U)-k(k-1) \int_{U}^{1} t^{k-2} \log (1-t) \mathrm{d} t\right] .
$$

(b) For all $k \geq 2, \sqrt{n} \hat{\Delta}_{k, \text { min }} \longrightarrow{ }_{d} \mathrm{~N}\left(0, \sigma_{k, \text { min }}^{2}\right)$, where

$$
\sigma_{k, \min }^{2}=\frac{(k-1)^{2}}{k^{2}(2 k-1)}
$$

(c) For all $k \geq 2, \sqrt{n} \hat{\Delta}_{k \text {,range }} \longrightarrow{ }_{d} \mathrm{~N}\left(0, \sigma_{k \text {,range }}^{2}\right)$, where

$$
\begin{aligned}
\sigma_{k, \text { range }}^{2}=\operatorname{Var}[ & \left(\frac{\mu_{k: k}^{e}-\mu_{1: k}^{e}}{k}-U^{k-1}+(1-U)^{k-1}\right) \log (1-U) \\
& \left.-(k-1) \int_{U}^{1}\left(t^{k-2}+(1-t)^{k-2}\right) \log (1-t) \mathrm{d} t\right] .
\end{aligned}
$$

Proposition 3 gives a result on the asymptotic behavior of the estimated discrepancies under the exponential distribution. Slight straightforward modifications of the proof yield the asymptotic distribution under a general distribution $F$ fulfilling mild regularity conditions. Alternative approaches to study the asymptotic behavior of S-Gini estimates can also be found in [14] and [16].

We highlight the result for the estimator $\hat{\Delta}_{2}$ because of its simplicity and usefulness in the subsequent applications:

Corollary 1. Let us assume that $H_{0}$ holds and that $n \rightarrow \infty$. We have that $\sqrt{n} \hat{\Delta}_{2} \longrightarrow{ }_{d} \mathrm{~N}(0,1 / 12)$.

By Proposition 3, it turns out that a suitable critical region with asymptotic level $\alpha$ is simply given by $R=\left\{\sqrt{n} \hat{\Delta}_{k, \bullet} / \sigma_{k, \bullet}>z_{\alpha}\right\}$ for any of the estimated discrepancies introduced above. However, it can be shown that $\hat{\Delta}_{k, \bullet}$ are biased estimators and that the biases, although asymptotically negligible, distort the significance levels of the tests when applied to finite samples. Fortunately, taking into account formula (3) for the expectation of the order statistics under the exponential distribution, it is straightforward to compute a bias correction of any of 
the discrepancies $\hat{\Delta}_{k, \bullet}$ under $H_{0}$. If we denote by $\mathrm{B}_{0}\left(\hat{\Delta}_{k, \bullet}\right)$ these bias corrections, the tests that we finally propose have the following critical region:

$$
R:=\left\{\sqrt{n}\left[\hat{\Delta}_{k, \bullet}-\mathrm{B}_{0}\left(\hat{\Delta}_{k, \bullet}\right)\right] / \sigma_{k, \bullet}>z_{\alpha}\right\} .
$$

Notice that the biases are $O\left(n^{-1}\right)$ and therefore the bias correction does not affect the asymptotic behavior of the estimators.

\subsection{Simulation study}

In order to examine the performance of the tests introduced above in terms of significance level and power we have carried out a simulation experiment. The design of the experiment mimics that of [8] so that the results can be compared.

Table 1 displays the empirical significance levels of six tests with the critical region (7) for the discrepancy measures $\hat{\Delta}_{2}$ and $\hat{\Delta}_{k, \bullet}$ with $k=3$ and $k=4$. Three values of $\alpha(0.01,0.05,0.10)$ and three different sample sizes $(20,50,100)$ have been considered. The experiment has been replicated 20000 times.

\begin{tabular}{|c|c|c|c|c|c|c|c|}
\hline & Test & $\hat{\Delta}_{2}$ & $\hat{\Delta}_{k, \max }$ & & $\hat{\Delta}_{k, \min }$ & & $\hat{\Delta}_{k, \text { range }}$ \\
\hline & $k$ & 2 & 3 & 4 & 3 & 4 & 4 \\
\hline \multirow{3}{*}{$n=20$} & $\alpha=0.01$ & 0.008 & 0.005 & 0.002 & 0.016 & 0.022 & 0.007 \\
\hline & $\alpha=0.05$ & 0.044 & 0.037 & 0.030 & 0.061 & 0.070 & 0.040 \\
\hline & $\alpha=0.10$ & 0.095 & 0.086 & 0.079 & 0.111 & 0.118 & 0.088 \\
\hline \multirow{3}{*}{$n=50$} & $\alpha=0.01$ & 0.009 & 0.006 & 0.005 & 0.012 & 0.016 & 0.007 \\
\hline & $\alpha=0.05$ & 0.048 & 0.044 & 0.040 & 0.056 & 0.060 & 0.045 \\
\hline & $\alpha=0.10$ & 0.098 & 0.095 & 0.091 & 0.104 & 0.108 & 0.095 \\
\hline \multirow{3}{*}{$n=100$} & $\alpha=0.01$ & 0.011 & 0.009 & 0.008 & 0.014 & 0.017 & 0.009 \\
\hline & $\alpha=0.05$ & 0.053 & 0.049 & 0.047 & 0.058 & 0.060 & 0.051 \\
\hline & $\alpha=0.10$ & 0.101 & 0.098 & 0.095 & 0.105 & 0.108 & 0.098 \\
\hline
\end{tabular}

Table 1: Empirical significance levels for different asymptotic tests, several values of $\alpha$ and several sample sizes $n$, 20000 replications.

The results of the simulation indicate that the true significance levels of the tests based on $\hat{\Delta}_{k \text {,max }}$ and $\hat{\Delta}_{k \text {,range }}$ seems to be slightly below the nominal one. However, the opposite happens for $\hat{\Delta}_{k, \min }$. This fact is more relevant for $n=20$ 
and $k=4$. Larger sample sizes $(n=50$ and $n=100)$ yield empirical significance levels fairly similar to the nominal ones.

\begin{tabular}{c|c|cc|cc|c} 
Test & $\hat{\Delta}_{2}$ & $\hat{\Delta}_{k, \max }$ & & $\hat{\Delta}_{k, \text { min }}$ & $\hat{\Delta}_{k, \text { range }}$ \\
\hline$k$ & 2 & 3 & 4 & 3 & 4 & 4 \\
\hline Exp(1) & 4.8 & 3.9 & 3.2 & 6.1 & 7.0 & 4.2 \\
$\mathrm{~W}(1.2)$ & 20.4 & 17.0 & 14.0 & 25.2 & 27.5 & 18.9 \\
$\mathrm{~W}(1.5)$ & 62.4 & 55.1 & 48.5 & 68.7 & 70.5 & 59.7 \\
$\mathrm{~W}(1.8)$ & 91.5 & 87.8 & 82.9 & 93.7 & 94.1 & 90.6 \\
$\mathrm{LFR}(0.5)$ & 17.2 & 14.9 & 12.6 & 19.9 & 20.6 & 15.8 \\
$\mathrm{LFR}(1.0)$ & 26.7 & 23.6 & 20.4 & 29.8 & 30.3 & 25.2 \\
$\mathrm{LFR}(2.0)$ & 40.1 & 36.5 & 32.0 & 43.3 & 42.9 & 38.3 \\
$\mathrm{LFR}(2.5)$ & 45.6 & 41.8 & 37.3 & 48.7 & 48.3 & 43.9 \\
LFR(3.0) & 49.6 & 45.8 & 41.0 & 52.6 & 52.2 & 47.7 \\
$\mathrm{G}(1.5)$ & 27.5 & 21.9 & 17.8 & 34.7 & 38.8 & 25.1 \\
$\mathrm{G}(2.0)$ & 59.7 & 50.2 & 42.4 & 69.9 & 74.3 & 56.7 \\
$\mathrm{G}(2.5)$ & 83.1 & 74.6 & 66.3 & 90.1 & 92.5 & 80.7 \\
Par(0.25) & 26.3 & 26.9 & 27.0 & 23.4 & 19.9 & 26.6 \\
Par(0.50) & 54.6 & 55.2 & 54.9 & 50.7 & 46.2 & 54.9 \\
Par(0.75) & 74.8 & 74.6 & 74.1 & 72.6 & 69.1 & 74.6 \\
$\operatorname{Par}(1.0)$ & 87.3 & 87.0 & 86.4 & 86.2 & 84.0 & 87.0 \\
Par(1.5) & 96.6 & 96.4 & 96.0 & 96.6 & 96.0 & 96.4
\end{tabular}

Table 2: Empirical power of different asymptotic tests, $\alpha=0.05, n=20,20000$ replications.

To assess power, together with the exponential (null hypothesis) we have also considered the following alternative distributions: Weibull, Gamma, Linear failure rate and Pareto distribution with scale parameter 1 and shape parameter $\theta$, denoted by $\mathrm{W}(\theta), \operatorname{LFR}(\theta), \mathrm{G}(\theta)$, and $\operatorname{Par}(\theta)$, respectively. The distribution functions are

$$
\begin{aligned}
F_{\mathrm{W}(\theta)}(x) & =1-\exp \left(-x^{\theta}\right), \quad x \geq 0, \theta>0, \\
F_{\mathrm{G}(\theta)}(x) & =\Gamma(\theta)^{-1} \int_{0}^{x} t^{\theta-1} e^{-t} \mathrm{~d} t, \quad x \geq 0, \quad \theta \geq 0, \\
F_{\mathrm{LFR}(\theta)}(x) & =1-\exp \left(-\left(x+\theta x^{2} / 2\right)\right), \quad x \geq 0, \theta \geq 0, \\
F_{\mathrm{Par}(\theta)}(x) & =1-(1+\theta x)^{-1 / \theta}, \quad x \geq 0, \quad \theta \geq 0,
\end{aligned}
$$

respectively. The six columns in Tables 2,3 and 4 display the empirical powers for the tests with the critical region (7) based on the discrepancy measures with 


\begin{tabular}{c|c|cc|cc|c} 
Test & $\hat{\Delta}_{2}$ & $\hat{\Delta}_{k, \max }$ & & $\hat{\Delta}_{k, \min }$ & $\hat{\Delta}_{k, \text { range }}$ \\
\hline$k$ & 2 & 3 & 4 & 3 & 4 & 4 \\
\hline $\operatorname{Exp}(1)$ & 4.9 & 4.5 & 4.0 & 5.4 & 5.9 & 4.6 \\
$\mathrm{~W}(1.2)$ & 42.4 & 38.2 & 34.8 & 46.2 & 47.3 & 40.8 \\
$\mathrm{~W}(1.5)$ & 96.8 & 95.4 & 93.6 & 97.4 & 97.3 & 96.5 \\
$\mathrm{~W}(1.8)$ & 100.0 & 100.0 & 99.9 & 100.0 & 100.0 & 100.0 \\
$\mathrm{LFR}(0.5)$ & 36.2 & 35.8 & 34.1 & 35.3 & 33.5 & 35.8 \\
$\mathrm{LFR}(1.0)$ & 59.0 & 58.7 & 57.0 & 56.6 & 53.2 & 58.7 \\
$\mathrm{LFR}(2.0)$ & 81.0 & 80.8 & 79.5 & 78.1 & 74.5 & 81.0 \\
$\mathrm{LFR}(2.5)$ & 85.9 & 85.7 & 84.5 & 83.7 & 80.2 & 85.8 \\
$\mathrm{LFR}(3.0)$ & 89.1 & 88.8 & 87.7 & 86.7 & 83.6 & 88.9 \\
$\mathrm{G}(1.5)$ & 57.6 & 50.7 & 45.3 & 65.9 & 69.4 & 55.3 \\
$\mathrm{G}(2.0)$ & 95.0 & 91.2 & 87.2 & 97.5 & 98.3 & 94.1 \\
$\mathrm{G}(2.5)$ & 99.8 & 99.4 & 98.7 & 99.9 & 100.0 & 99.7 \\
$\operatorname{Par}(0.25)$ & 47.9 & 49.6 & 50.2 & 41.2 & 35.5 & 49.0 \\
$\operatorname{Par}(0.50)$ & 85.7 & 86.2 & 86.2 & 81.9 & 77.0 & 86.1 \\
$\operatorname{Par}(0.75)$ & 97.5 & 97.6 & 97.5 & 96.6 & 95.2 & 97.5 \\
$\operatorname{Par}(1.0)$ & 99.6 & 99.6 & 99.6 & 99.5 & 99.3 & 99.6 \\
$\operatorname{Par}(1.5)$ & 100.0 & 100.0 & 100.0 & 100.0 & 100.0 & 100.0
\end{tabular}

Table 3: Empirical power of different asymptotic tests, $\alpha=0.05, n=50,20000$ replications.

$k=2, k=3$ and $k=4$. The asymptotic level of the tests is $\alpha=0.05$. Sample sizes are $n=20, n=50$ and $n=100$. The experiment has been replicated 20000 times.

In terms of power there is not any test that is clearly better than the others. The observed differences in power can be accounted for the fact that the actual significance levels are not exactly equal to the nominal value $\alpha=0.05$. The value of $k$ does not seem to have a systematic effect on the power either although $k=2$ tends to give better results than $k=3$ and $k=4$. The empirical powers in Tables 2 and 3 are similar to those reported in [8] (in that paper, the case $n=100$ is not considered).

As a conclusion we recommend the use of the test based on $\hat{\Delta}_{2}$ since it gives results comparable to the other tests and exhibits three worthy advantages: a) the empirical level practically coincides with the nominal one for the sample sizes considered in the study, b) the expression of the asymptotic variance (see Corollary 1 ) is remarkably simple and makes this test straightforward to implement and use. 
Finally, c) this test has a clear interpretation in reliability theory as it was pointed out in Remark 1 above.

\begin{tabular}{c|c|cc|cc|c} 
Test & $\hat{\Delta}_{2}$ & $\hat{\Delta}_{k, \max }$ & & $\hat{\Delta}_{k, \min }$ & $\hat{\Delta}_{k, \text { range }}$ \\
\hline$k$ & 2 & 3 & 4 & 3 & 4 & 4 \\
\hline $\mathrm{Exp}(1)$ & 5.0 & 4.7 & 4.5 & 5.3 & 5.7 & 4.8 \\
$\mathrm{~W}(1.2)$ & 68.2 & 64.6 & 60.9 & 71.3 & 71.4 & 67.2 \\
$\mathrm{~W}(1.5)$ & 100.0 & 99.9 & 99.9 & 99.9 & 100.0 & 100.0 \\
$\mathrm{~W}(1.8)$ & 100.0 & 100.0 & 100.0 & 100.0 & 100.0 & 100.0 \\
$\mathrm{LFR}(0.5)$ & 61.4 & 62.4 & 62.0 & 56.2 & 51.3 & 61.8 \\
$\mathrm{LFR}(1.0)$ & 87.2 & 88.2 & 88.2 & 82.9 & 78.3 & 87.7 \\
$\mathrm{LFR}(2.0)$ & 98.0 & 98.3 & 98.2 & 96.6 & 94.4 & 98.2 \\
$\mathrm{LFR}(2.5)$ & 99.2 & 99.3 & 99.4 & 98.4 & 97.1 & 99.3 \\
$\mathrm{LFR}(3.0)$ & 99.6 & 99.7 & 99.7 & 99.0 & 98.3 & 99.6 \\
$\mathrm{G}(1.5)$ & 85.8 & 80.2 & 75.3 & 90.7 & 92.3 & 83.9 \\
$\mathrm{G}(2.0)$ & 99.9 & 99.7 & 99.4 & 100.0 & 100.0 & 99.9 \\
$\mathrm{G}(2.5)$ & 100.0 & 100.0 & 100.0 & 100.0 & 100.0 & 100.0 \\
Par(0.25) & 69.7 & 71.6 & 72.3 & 61.8 & 54.3 & 71.1 \\
Par(0.50) & 98.2 & 98.3 & 98.3 & 97.0 & 95.2 & 98.3 \\
Par(0.75) & 100.0 & 100.0 & 100.0 & 99.9 & 99.8 & 100.0 \\
Par(1.0) & 100.0 & 100.0 & 100.0 & 100.0 & 100.0 & 100.0 \\
Par(1.5) & 100.0 & 100.0 & 100.0 & 100.0 & 100.0 & 100.0
\end{tabular}

Table 4: Empirical power of different asymptotic tests, $\alpha=0.05, n=100,20000$ replications.

\section{Appendix: Proofs}

Proof of Proposition 1: First, we note that if $X$ is exponentially distributed with mean $\mu$, it is easy to check that, for $k, p \geq 1,0 \leq m \leq k$ and $0 \leq l \leq p$, we have

$$
S_{m: k}(X)=\mu m\left(1+\mu_{k-m: k}^{e}\right), \quad s_{l: p}(X)=\mu\left(l-(p-l) \mu_{l: p}^{e}\right),
$$

where $\mu_{i: j}^{e}$ is defined in (3). Thus, condition (a) obviously implies (b).

Now, let us assume that $X$ is an HNBUE variable (the proof for HNWUE variables runs in a similar fashion) and that (b) holds for some fixed $k \geq 2$ and $1 \leq m<k$ or for some $p \geq 2$ and $1 \leq l<p$. We obviously have,

$$
S:=S_{m: k}(X)-s_{l: p}(X)=S_{m: k}(X)+S_{p-l: p}(X)-p \mathrm{E} X
$$


By [1, eq. (5.2.8)], we have, for all $j \geq 1$ and $1 \leq i \leq j$,

$$
\mathrm{E} X_{i: j}=j\left(\begin{array}{c}
j-1 \\
i-1
\end{array}\right) \int_{0}^{1} F^{-1}(t) t^{i-1}(1-t)^{j-i} \mathrm{~d} t
$$

Therefore, by (10), we obtain

$$
S=\int_{0}^{1} F^{-1}(t) \omega(t) \mathrm{d} t
$$

where

$$
\begin{aligned}
\omega(t):= & k \sum_{i=k+1-m}^{k}\left(\begin{array}{c}
k-1 \\
i-1
\end{array}\right) t^{i-1}(1-t)^{k-i} \\
& +p \sum_{i=l+1}^{p}\left(\begin{array}{c}
p-1 \\
i-1
\end{array}\right) t^{i-1}(1-t)^{p-i}-p \\
= & k \mathrm{P}\left(\beta_{k-m, m} \leq t\right)+p\left[\mathrm{P}\left(\beta_{l, p} \leq t\right)-1\right],
\end{aligned}
$$

where $\beta_{i, j}$ stands for a $\operatorname{Beta}(i, j)$ random variable for $1 \leq i \leq j$ and $\beta_{0, j}$ is degenerate at 0 . For the last step in the equality above we have used [7, eq. (3.37)]. $X$ is HNBUE with mean $\mu$, and hence $X \leq_{c x} Y$, where $Y$ is an exponential random variable with mean $\mu$. From (9) and (11), we have that the condition (b) can be expressed by $\Delta_{\omega}(X, Y)=0$, where $\omega \in \mathcal{I}^{*}$ is given in (12). Therefore, a direct application of Theorem 1 leads to $X={ }_{s t} Y$ exponential with mean $\mu$ and the proof of the corollary is completed.

Proof of Proposition 3: (a) The estimator $\hat{\Delta}_{k \text {,max }}$ given in (6) is asymptotically equivalent to the simpler one

$$
\tilde{\Delta}_{k, \max }:=\mu_{k: k}^{e}-\frac{1}{\bar{X}} \frac{k}{n} \sum_{i=1}^{n}\left(\frac{i}{n}\right)^{k-1} X_{i: n} .
$$

To see this notice that by the mean value theorem, for $i=1, \ldots, n$ there exists $\theta_{i} \in((i-1) / n, i / n)$ such that

$$
\left|\hat{\Delta}_{k, \max }-\tilde{\Delta}_{k, \max }\right| \leq \frac{1}{\bar{X}} \frac{k}{n} \sum_{i=1}^{n}\left[\left(\frac{i}{n}\right)^{k-1}-\theta_{i}^{k-1}\right] X_{i: n} \leq \frac{k(k-1)}{n} \frac{\sum_{i=1}^{n} X_{i: n}}{n}
$$


and since $X$ is integrable the last expression is $O_{p}(1 / n)$.

The estimator $\tilde{\Delta}_{k, \max }$ can be written in the following way:

$$
\tilde{\Delta}_{k, \max }=\frac{1}{\bar{X}} \sum_{i=1}^{n} J_{k}(i / n) X_{i: n}
$$

where $J_{k}(u):=\mu_{k: k}^{e}-k u^{k-1}$. Finally, we apply [11, Theorem 2.1] with $H(u):=u$ and $J(u):=J_{k}(u)$ to obtain the result.

(b) Following the same lines of the proof of part (a) it is possible to show that $\hat{\Delta}_{k \text {,min }}$ is asymptotically normal with the following asymptotic variance:

$$
\begin{aligned}
\sigma_{k, \text { min }}^{2}=\operatorname{Var}[ & \left(k(1-U)^{k-1}-\mu_{1: k}^{e}\right) \log (1-U) \\
& \left.+k(k-1) \int_{U}^{1}(1-t)^{k-2} \log (1-t) \mathrm{d} t\right]
\end{aligned}
$$

The integral in the above expression can be worked out:

$$
\int_{U}^{1}(1-t)^{k-2} \log (1-t) \mathrm{d} t=\frac{(1-U)^{k-1}}{k-1}\left((1-U)^{k-1}-\frac{1}{k-1}\right)
$$

Replacing this result in (13) yields:

$$
\begin{aligned}
\sigma_{k, \text { min }}^{2} & =\operatorname{Var}\left(\frac{1}{k} \log (U)-\frac{k}{k-1} U^{k-1}\right) \\
& =\frac{1}{k^{2}}+\left(\frac{k}{k-1}\right)^{2} \operatorname{Var}\left(U^{k-1}\right)-2 \frac{k^{2}}{k-1} \operatorname{Cov}\left(\log (U), U^{k-1}\right) \\
& =\frac{(k-1)^{2}}{k^{2}(2 k-1)}
\end{aligned}
$$

(c) The proof of this part is totally analogous to that of part (a).

\section{Acknowledgments}

The authors are thankful to an anonymous referee for pointing out reference [6] and correcting a mistake in the previous version of this manuscript. 


\section{References}

[1] B.C. Arnold, N. Balakrishnan and H.N. Nagaraja (1992). A First Course in Order Statistics, Wiley, New York.

[2] R.E. Barlow and F. Proschan (1975). Statistical Theory of Reliability and Life Testing: Probability Models. Holt, Rinehart and Winston, New York.

[3] A.P. Basu and S.N.U.A. Kirmani (1986). Some results involving HNBUE distributions, J. Appl. Prob. 23, 1038-1044.

[4] J.R. Berrendero and J. Cárcamo (2008). Tests for second order stochastic orders based on mean order statistics, submmited for publication. Available at http://www.uam.es/joser.berrendero/articulos/test-eco3.pdf

[5] J. Cai (1994). Characterizations of life distributions by moments of extremes and sample mean, J. Appl. Prob. 31, 148-155.

[6] M.I. Hendi, H. Al-Nachawati, M. Montasser and I.A. Alwasel (1998). An exact test for HNBUE class of life distributions, J. Statist. Comput. Simul. 60, 161-275.

[7] N.L. Johnson, S. Kotz and A.W. Kemp (1992). Univariate Discrete Distributions, 2nd ed. Wiley, New York.

[8] B. Klar (2000). A class of tests for exponentiality against HNBUE alternatives, Statist. Probab. Lett. 47, 199-207.

[9] B. Klefsjö (1983). Testing exponentiality against HNBUE. Scand. J. Statist. 10, $65-75$.

[10] P.J. Lambert (1993). The distribution and redistribution of income, a mathematical analysis. Manchester University Press, Manchester, 2nd edition.

[11] D. Li, M.B. Rao and R.J. Tomkins (2001). The law of the iterated logarithm and central limit theorem for L-Statistics, J. Multivariate Anal. 78, 191-217. 
[12] A. Müller and D. Stoyan (2002). Comparison Methods for Stochastic Models and Risks, Wiley, New York.

[13] M. Shaked and J.G. Shanthikumar (2006). Stochastic Orders, Springer.

[14] K. Xu (2000). Inference for generalized Gini indices using the iteratedbootstrap method. J. Bus. Econom. Statist. 18, 223-227.

[15] S. Yitzhaki (1983). On the extension of the Gini inequality index. Internat. Econom. Rev. 24, 617-628.

[16] R. Zitikis and J.L. Gastwirth (2002). The asymptotic distribution of the SGini index. Aust. N. Z. J. Stat. 44, 439-446. 